\title{
IL DIACONATO NELLA RIFLESSIONE TEOLOGICA DEI RIFORMATORI MARTIN LUTERO, HULDRYCH ZWINGLI E GIOVANNI CALVINO
}

DOI: http://dx.doi.org/10.12775/TiCz.2020.007

Streszczenie. Diakonat w teologicznej refleksji reformatorów Marcina Lutra, Huldrycha Zwingli i Giovanniego Calvino. Protestanccy reformatorzy z XVI wieku Luter, Zwingli i Kalwin nie przywiązują większej wagi do tożsamości i posługi diakona w ich refleksji teologicznej. Zdaniem Lutra diakoni są powołani do poświęcenia swoich sił i czasu na służbę, która przede wszystkim polega na tym, aby Słowo Boże żyło w świecie nie tylko za pośrednictwem przepowiadania, ale także poprzez czyny. Diakonat zdecydowanie różni się od posługi Słowa i ma znaczenie drugorzędne w porównaniu $z$ nią. Zwingli utrzymuje, że diakoni, podobnie jak słudzy opisani w Dziejach Apostolskich, są w jakiejś mierze sługami Słowa. Dlatego oprócz opieki nad chorymi, zaspakajania potrzeb ubogich są zobowiązani do pełnienia posługi Słowa. Diakoni podejmują się charytatywnego zadania wobec całego społeczeństwa. Kalwin określa i rozwija koncepcję diakonatu, szczególnie w kontekście teorii czterech posług. On jest tym, który chce pójść dalej w instytucjonalnym przywróceniu posługi kościelnej diakona. Zdaniem Kalwina diakonat powinien odznaczać się czteroma cechami: być posługą specyficzną, konsekrowana o charakterze permanentnym, charytatywno-społeczna oraz kultowa.

Słowa kluczowe: Kościół; diakonia; diakon; diakonat; posługa kościelna; posługa Słowa; dzieła miłosierdzia; pastorostwo; prezbiterat; reformacja protestancka; reformatorzy; ubóstwo. 
Abstract. The diaconate in the theological considerations of the Reformers Martin Luther, Huldrych Zwingli and John Calvin. The Reformers of the Protestant church from the XVI century, namely Luther, Zwingli and Calvin, did not pay much attention, in their theological reflections, to the identity and ministry of the deacon. According to Luther, deacons were called to dedicate their efforts and time to a ministry whose primary objective was to bring to life the Word of God in the world, not only through their words and discourses, but also through their actions. The diaconate is distinct from the ministry of the Word and is secondary to it. In Zwingli's view, deacons, similar to the servants described in the Acts of the Apostles, form part of the ministry of the Word. Those who care for the sick, who visit them, who concern themselves with solidarity and provision for the poor, are also ministers of the Word. For Zwingli, these take on a charitable role towards society as a whole. Calvin developed a more precise understanding of the diaconate with his theory of the four ministries. He went furthest in his attempts at an institutional restoration of the ecclesial ministry of the diaconate. Calvin held the view that the diaconate required four characteristics: specific (along with other ministries, especially pastoral), permanent and consecrated, charitable and cultural.

Keywords: Church; Diaconia; Deacon; Diaconate; Ecclesial Ministry; Ministry of the Word; Works of Charity; Pastoral Work; Presbyterate; Protestant Reform; Reformers; Poverty.

\section{INTRODUZIONE}

Il ministro ordinato diacono, è una realtà antichissima, già presente nella Chiesa apostolica e di grande rilevanza nei primi secoli.

Cristo, in modo diretto, istituì solamente il Collegio apostolico. $\mathrm{Ma}$ già gli Atti degli Apostoli attestano che essi stessi avvertirono il bisogno di avere collaboratori cui affidare il servizio delle mense. Una lunga tradizione vede in questo fatto l'istituzione del ministero diaconale.

La Chiesa, nell'arco dei secoli, ha sempre inteso l'istituzione della pienezza del ministero ordinato sacerdotale negli Apostoli congiunta con la potestà di individuare altre funzioni particolari.

Sta il fatto che il diaconato, importantissimo nei primi secoli, decadde progressivamente, riducendosi a semplice grado temporaneo del cursus ecclesiastico, sino a che il Concilio Vaticano II ha deliberato di riproporlo come ministero ordinato permanente ${ }^{1}$.

\footnotetext{
${ }^{1}$ Cf. R. Selejdak, Diakonat stały w świetle dokumentów Soboru Watykańskiego II,
} 
Lo studio presente cerca di esaminare la tematica del diaconato nella riflessione teologica dei tre grandi Riformatori della Chiesa protestante del XVI secolo: Martin Lutero, Huldrych Zwingli e Giovanni Calvino.

\section{MARTIN LUTERO (1483-1546)}

La riflessione teologica del Riformatore Martin Lutero si sviluppa a partire dalla sua iniziale considerazione secondo cui chi dice diaconia dice opere. La teologia della salvezza mediante le opere è il bersaglio privilegiato della sua azione riformatrice.

Un'opera non può mai essere meritoria, e una diaconia teologicamente ben compresa non potrà mai essere legata ai meriti o alle indulgenze: un'opera non può essere, secondo Lutero, che il frutto della fede, risultando, perciò, secondaria rispetto a quest'ultima ${ }^{2}$.

Lopera considerata come meritoria e la fiducia posta nelle indulgenze possono distogliere diabolicamente il credente dal suo dovere di carità.

Lutero insiste su questo punto sin dalle novantacinque tesi del 1517:

Bisogna insegnare ai cristiani che chi dà ai poveri o presta a chi è nel bisogno, agisce meglio che se avesse comprato delle indulgenze. Infatti, mediante l'opera della carità, la carità cresce, e l'uomo è reso migliore, mentre mediante le indulgenze non è reso migliore, ma soltanto è più libero dalla pena. Bisogna insegnare ai cristiani che chi vede un povero e, trascuratolo, acquista delle indulgenze, chiama su di sé non le indulgenze del papa, ma la collera di Dioº

posoborowego Urzędu Nauczycielskiego Kościoła i narodowych «Rationes institutionis diaconorum permanentium», Warszawa 2010, pp. 34-74.

${ }^{2}$ Cf. G. Hammann, Storia del diaconato. Dal cristianesimo delle origini ai riformatori protestanti del XVI secolo, Magnano 2004, p. 226; P. Meroni, Il diacono. Segno di rinnovamento della Chiesa. Presenza nella famiglia e nella vita della società, Roma 2010, p. 77.

${ }^{3}$ M. Luther, Tesi 43-45, in: Werke. Kritische Gesamtausgabe, Weimar 1883, p. 235; idem, Oeuvres, Genève 1957, p. 109. 
In questi tesi che stanno per avviare l'azione riformatrice, la teologia della salvezza è alla base del dibattito. Attraverso la questione del peccato e della sua redenzione è coinvolta l'autenticità redentrice della fede come relazione con Cristo crocifisso. La diaconia è al centro della questione in quanto pratica caritativa. Per Lutero, essa è sempre tale, se vuole essere conforme al Vangelo.

In quanto espressione di carità, essa riguarda effettivamente il tema della salvezza e dei frutti della fede. Mentre, invece, non si verifica necessariamente la stessa cosa per quanto concerne il ministero ordinato di diacono. Secondo la teologia dei ministeri propria dei Riformatori protestanti, e in particolare di Lutero, il ministero specifico di cui la Chiesa ha bisogno, è quello della Parola, cioè il ministero pastorale.

Questo ministero è il solo di cui la comunità cristiana abbia specificamente bisogno per vivere e per continuare a svilupparsi. Esso è, dunque, il solo rispetto al quale la comunità debba necessariamente ordinare certe persone. Per Lutero, non cè differenza sostanziale di stato tra il clero e i laici: l'ordinazione ministeriale non conferisce nessuna qualità speciale alle persone che, conseguentemente, si distinguerebbero nei confronti degli altri battezzati.

Lunica "ordinazione" che introduce i cristiani in uno stato particolare, lessere in Cristo, è il battesimo.

I ministeri, al contrario - e tra loro, secondo Lutero, anche i diaconi - sono semplicemente consacrati, dalla comunità ecclesiale, per dedicare le loro forze e il loro tempo al ministero che in primo luogo consiste nel far vivere la Parola di Dio nel mondo mediante la predicazione e i sacramenti ${ }^{4}$. Ora, nella misura in cui questa predicazione della Parola deve avvenire non soltanto con le parole e con i discorsi, ma anche con le azioni, il ministero della Chiesa assume una connotazione diaconale e può richiedere alloccasione un ministero di diacono.

Lutero indica il riferimento al diaconato in un passo del trattato La cattività babilonese della chiesa una delle tre grandi opere pubblicate nel 1520.

${ }^{4}$ Cf. G. Miegge, Lutero. L'uomo e il pensiero fino alla Dieta di Worms, Torino 2001, p. 64. 
In tale passo si trovano i temi della riforma indispensabile della Chiesa, del vero ministero della Chiesa affidato a tutti i battezzati (il "sacerdozio universale"), del ministero specifico della Parola affidato mediante ordinazione ai presbiteri o ai vescovi (Lutero non distingue tra i due) e del ministero di diacono.

Lutero afferma:

Qual è mai l'ignominia che la chiesa di Dio ha contratto dai suoi mostri sacerdotali! Dove sono i vescovi o i preti che conoscono l'Evangelo, per non dire: chi lo predica? Perché dunque si vantano di essere preti? Perché vogliono essere considerati come più santi e migliori, in possesso di un potere più grande di quello degli altri cristiani? [...] L'incarico del prete è di predicare: se non lo fa, non è più prete così come l'immagine di un uomo non è l'uomo. È proprio del vescovo ordinare simili preti capaci solo a ripetere preghiere? O forse consacrare chiese e campane? O cresimare i fanciulli? No, queste cose possono essere fatte anche da un diacono o da un laico qualsiasi. È il ministero della Parola che fa il prete e il vescovo ${ }^{5}$.

Per Lutero, solo la potenza teologica della Parola poteva riformare la chiesa, poiché tutti gli altri tentativi ecclesiologici (specialmente quelli dei concili) avevano fallito. Di qui, quest'insistenza protestante che, in retrospettiva, può sembrare esagerata sul ministero della Parola. Per riformare la chiesa dell'epoca, bisognava formare e consacrare delle persone per questa funzione, non per conferire loro un carattere o uno stato speciale, diverso da quello del popolo dei battezzati, ma per affidare loro questa responsabilità essenziale di proclamare, di spiegare, di interpretare, di far comprendere la vita e il mondo alla luce di questa Parola.

Le affermazioni di Lutero sui ministeri sono sostenute da una duplice preoccupazione riformatrice: da una parte la preoccupazione di restituire al popolo di Dio, cioè a ogni battezzato la sua responsabilità ecclesiale; dall'altra parte responsabilizzare il popolo dei laici per mezzo della predicazione alimentata dalla Parola biblica tramite dei ministri consacrati a questo compito.

${ }^{5}$ M. Luther, De captivitate Babylonica Ecclesiae, in: WA VI, p. 566; idem, Oeuvres II, pp. 251-252. 
Il compito di diacono, d'altra parte è ecclesialmente legato al ministero della Parola. Effettivamente, da un punto di vista storico e secondo At $6,1-7$, dice Lutero, il ministero dei diaconi è derivato dal sovraccarico dei ministri della predicazione apostolica, sovraccarico dovuto al servizio delle "vedove", ossia dei poveri. Dunque, per lui, il diaconato ha fondamentalmente una responsabilità in ordine alla carità ${ }^{6}$.

Lutero in questo stesso testo definisce più positivamente la sua concezione dei tre ministeri: quello del laico (la responsabilità di battezzato in questo sacerdozio universale), il ministero della Parola (il pastorato) e il ministero del servizio di carità (il diaconato):

Chiunque si dichiara cristiano sappia con certezza che siamo tutti ugualmente sacerdoti, cioè che abbiamo lo stesso potere a riguardo della Parola e di ogni sacramento, benché a nessuno sia permesso di usare senza il consenso della comunità o la chiamata di un superiore (infatti, fino al momento in cui è chiamato, nessuno può arrogarsi di sua propria testa ciò che è comune a tutti). Si sappia che per questo motivo, il sacramento dell'ordine, se esso è qualche cosa, non è altro che una certa maniera di chiamare qualcuno al ministero ecclesiastico e che il sacerdozio propriamente non è che il ministero della Parola. Dico della Parola, non della Legge, ma dell'Evangelo. Il diaconato, da parte sua, non consiste nella lettura del vangelo o dellepistola, come vuole l'uso attuale, ma nel distribuire i soccorsi della chiesa ai poveri, affinché i preti siano sollevati dal peso degli affari temporali e più liberamente si applichino alla preghiera e alla Parola. In At 6 si legge che i diaconi furono istituiti effettivamente con questa intenzione ${ }^{7}$.

Anche qui risulta che il ministero diaconale non ha nella Chiesa lo stesso fondamento teologico del pastorato e che dunque non potrebbe avere funzioni simili a questo. La diaconia non consiste nella lettura dell'Evangelo e dell'epistola, dice Lutero. Dicendo ciò, contesta quel che rimane del significato cultuale che la Chiesa medievale ha conservato delle Chiese delle origini e antiche, senza che esse siano riuscite tuttavia a mantenere il legame originario tra il culto domenicale e la vita quoti-

${ }^{6}$ Cf. G. Hammann, Storia del diaconato. Dal cristianesimo delle origini ai riformatori protestanti del XVI secolo, p. 229.

${ }^{7}$ M. Luther, De captivitate Babylonica Ecclesiae, in: WA VI, p. 566; idem, Oeuvres II, pp. 251-252. 
diana della comunità. Se Lutero giustamente critica questo carattere cultualmente striminzito del diaconato tradizionalmente ordinato, egli non gli concede evidentemente nessun ruolo liturgico rinnovato, come quello che un tempo, nelle Chiese antiche, era rappresentato dall'offertorio e dal valore caritativo dell'eucaristia. Lutero certamente attribuisce al diaconato un fondamento specifico, ma quanto a importanza teologica lo subordina al ministero della Parola; esso è secondario rispetto a quest'ultimo. Nel suo fondamento (come, d'altra parte, nella pratica), il ministero diaconale è ben distinto da quello della Parola, il solo essenziale alla specificità evangelica della Chiesa. Al ministero della Parola viene data una qualificazione spirituale ("della Parola, non della Legge, ma dell'Evangelo"), al ministero di diacono una qualificazione materiale ("sollevare dal peso degli affari temporali").

Il diaconato non esiste e non trova la sua qualificazione spirituale se non tramite il pastorato, di cui segue le tracce, come i frutti seguono la giustificazione del credente mediante la sola fede. La fede produce le opere. Allo stesso titolo, il ministero della Parola (l'Evangelo predicato) produce il suo inevitabile complemento in questo ministero successivo che è il ministero diaconale. Fede e amore vanno di pari passo; ugualmente il pastorato e il diaconato:

Ecco la regola di cui bisognerà ricordarsi: tutti i beni che noi abbiamo da Dio si diffondano dall'uno all'altro e siano messi in comune; ciascuno si rivesta del suo prossimo e si prenda cura di lui come di se stesso. Di questi beni, Cristo è stato e resta la fonte da dove fluiscono in noi: egli si è rivestito di noi e si è preso cura di noi come se fosse quello che noi siamo. Da noi devono fluire in coloro che ne hanno bisogno; così davanti a Dio, io devo porre la mia fede per coprire i peccati del mio prossimo e implorare il loro perdono, prendendoli su di me, con il loro tormento e la loro schiavitù, come se fossero miei appunto, proprio come Cristo ha fatto per noi. Ecco il vero amore, ecco la pura regola della vita cristiana. Ma essa è vera e pura soltanto dove la fede è vera e pura [...]. Concludiamo dicendo che il cristiano non vive in se stesso, ma vive in Cristo e nel suo prossimo. Altrimenti non è cristiano. Egli vive al di sopra di se stesso in Dio mediante l'amore, egli è abbassato al di sotto di se stesso nel suo prossimo. Eppure egli rimane sempre in Dio e nel suo amore ${ }^{8}$.

${ }^{8}$ Idem, La libertà del cristiano, in: WA VII, p. 69; Oeuvres II, pp. 300-301. 
Secondo Lutero, non si potrebbero distinguere meglio i rispettivi fondamenti del pastorato (la fede) e del diaconato (la carità) e il loro posto specifico nella teologia dei ministeri. Essi sono certamente complementari, entrambi fondati nella Scrittura, ordinati dalla comunità, ma successivi e di qualificazione differente. La fede e il ministero della Parola che le è collegato sono primordiali a tal punto che, in circostanze eccezionali, per esempio quando non è presente alcun altro ministro, il diacono può sopperire all'assenza del pastorato e divenire predicatore dell'Evangelo. Tale è il caso di Stefano o di Filippo nel libro degli Atti, i quali non avevano in nessun modo ricevuto una vocazione per questo compito. Il diaconato sarebbe dunque destinato alla predicazione? No, dice Lutero, solo in casi eccezionali che non potrebbero giustificare la confusione tra i due ministeri poiché, se questi due "diaconi" hanno potuto nella fattispecie andare oltre la loro vocazione, questo non è avvenuto in quanto ministri ordinati, ma in quanto semplici cristiani, chiamati a proclamare l'Evangelo come ogni altro battezzato in simili circostanze. A tale riguardo ha scritto nel trattato intitolato "Secondo la Scrittura uniassemblea o comunità cristiana ha il diritto e la facoltà di giudicare ogni dottrina e di chiamare, insediare e destituire i predicatori":

Ancora una volta, dunque, è cosa certa che un cristiano non ha soltanto il diritto e la facoltà di insegnare la parola di Dio, ma ha anche il dovere di farlo sotto la pena di perdere la sua anima e di incorrere nello sdegno di Dio. Tu dici allora: «Ma come? Se egli non è chiamato a questo, non può arrischiarsi a predicare, come tu hai spesso insegnato!». Risposta: tu devi assegnare al cristiano un compito differente a seconda dei luoghi ove egli si trova. In primo luogo: se egli si trova in un luogo in cui non cè nessun cristiano, non ha bisogno di una chiamata diversa da quella della sua stessa condizione di cristiano, in virtù della quale egli è interiormente chiamato da Dio e sa di essere unto di lui; egli ha il dovere di predicare in quel luogo e di insegnare l'Evangelo ai pagani e agli infedeli, per amore fraterno, anche se nessuno lo ha chiamato a ciò. È quello che ha fatto santo Stefano (At 6 e 7) [...] e quello che ha fatto Filippo, il diacono compagno di Stefano (At 8), cui il ministero della predicazione non era stato affidato. Così fece anche Apollo (At 18). Infatti, in un caso di genere, il cristiano, a motivo dellamore fraterno, guarda la disperazione delle povere anime perdute e non aspetta che gli sia dato un ordine o una lettera di nomina da parte dei principi o dei vescovi. Infatti la necessità 
elimina tutte le leggi e non conosce legge. Allo stesso modo l'amore deve venire in aiuto là dove non cè nessuno che aiuti o che possa aiutare?

In questo stesso trattato, Lutero conferma questo fondamento del ministero diaconale. Egli ripete che il diaconato è "di assai minor importanza" rispetto al pastorato, ma che tuttavia, per analogia con la pratica dei tempi apostolici, è un ministero ordinato dalla comunità e per l'imposizione delle mani:

Sempre in Atti 6, leggiamo che per un ministero di assai minore importanza, gli apostoli stessi non osavano costituire nessuno diacono senza che la comunità lo sapesse e lo volesse; è la comunità invece che scelse e chiamò i sette diaconi e gli apostoli li confermarono. Ora, se per un tale ministero che consisteva nel distribuire un nutrimento temporale gli apostoli non avevano il diritto di stabilire qualcuno di loro propria autorità, come avrebbero potuto permettersi di propria iniziativa di imporre qualcuno per il ministero superiore della predicazione senza che la comunità lo avesse saputo, lo avesse voluto e chiamato? ${ }^{10}$.

Ciò vuol dire che il ministero di diacono, dal momento che deve essere istituito e ordinato, deve esserlo allo stesso titolo del ministero della Parola. Secondo questo testo, l'ordinazione (la consacrazione) di diaconi da parte della comunità ecclesiale non pone nessun problema, se non quello relativo alle disposizioni ecclesiastiche.

Lutero in una predica del 1522 su At 6, 1-7, pronunciata il giorno di santo Stefano, il 26 dicembre, in memoria del primo dei "diaconi", affronta l'aspetto caritativo del diaconato:

È vero che Stefano ha spesso assolto la funzione di intendente o di curatore verso i cristiani per distribuire i beni materiali a coloro che ne avevano bisogno [...]. Di questa funzione non resta che quella piccola parte garantita da alcune monache, priori, dai patroni di ospizio o dai curatori dei poveri [...]. Ecco il compito che dovrebbero assumere questi ministri delle epistole e dei vangeli, non i consacrati e i tosati [...]

9 Idem, Secondo la Scrittura uniassemblea o comunità cristiana ha il diritto e la facoltà di giudicare ogni dottrina e di chiamare, insediare e destituire i predicatori, in: WA XI, p. 412; Oeuvres IV, pp. 85-86.

${ }^{10}$ Ibidem, in: WA XI, p. 414; Oeuvres IV, pp. 87-88. 
ma la maggior parte dei laici e l'uomo di fede, questi dovrebbe tenere il registro e avere la responsabilità della casa comune, per distribuire là dove necessario. Questo è il ministero proprio di santo Stefano, che non si è mai sognato la lettura dell'epistola o dell'Evangelo, né la tonsura o le cerimonie. Queste cose non sono che invenzioni umane ${ }^{11}$.

Lutero mette in evidenza l'aspetto caritativo del diaconato contro l'impoverimento strettamente liturgico che esso ha conosciuto nel Medioevo. Ora, per Lutero, il ministero di Stefano nel libro degli Atti è senza esitazione, proprio il tipo del ministero del diacono. Questo ministero, nella comunità primitiva, non avrebbe avuto niente a che vedere con un ministero cultuale, poiché questo diacono - tipo non si sarebbe mai sognato un tale ministero, né una qualsiasi cerimonia liturgica. Per il Riformatore, la forma cultuale del ministero diaconale al momento della messa non può essere che una deviazione, una "invenzione umana”. Perciò da abolire! E la riforma del ministero diaconale, alla luce della Scrittura (cioè di At 6), deve passare attraverso la soppressione di questo aspetto clericale, falsamente cultuale, che gli è stato conferito da una tradizione deviante ${ }^{12}$.

\section{HULDRYCH ZWINGLI (1484-1531)}

Secondo gli storici Zwingli al riguardo del diaconato sostiene una dottrina e una pratica originali in confronto agli altri Riformatori protestanti ${ }^{13}$. Spingendosi assai oltre rispetto a Lutero, egli riorganizza il

11 M. Luther, in: WA X/I, pp. 262-263.

${ }^{12}$ Cf. G. Hammann, Storia del diaconato. Dal cristianesimo delle origini ai riformatori protestanti del XVI secolo, p. 237.

${ }^{13}$ Cf. W. Köhler, Armenpflege und Wohltätigkeit in Zürich zur Zeit Ulrich Zwinglis, Zürich 1919; A. Denzler, Geschichte des Armenwesens im Kanton Zürich im 16. und 17. Jahrhundert, Zürich 1929; W. Bernoulli, Von der reformierten Diakonie der Reformationszeit, in: Das diakonische Amt der Kirche, a cura di H. Krimm, Stuttgart 1965², pp. 197-202; G. W. Locher, Die Zwinglische Reformation im Rahmen der europäischen Kirchengeschichte, Göttingen-Zürich 1979; M. Lienhard, Laction et la doctrine de Huldrych Zwingli, in: Histoire du christianisme, a cura di G. Livet, F. Rapp, vol. VII, Strasbourg 1982, pp. 771-787; B. Hamm, Zwinglis Reformation der Freiheit, Neukirchen 1988; 
diaconato secondo un vero programma politico e sociale della città cristiana. Per lui (come per gli altri Riformatori, ma in maniera ancora più radicale) la diaconia cristiana deve essere solo caritativa. Pertanto egli può essere considerato come il padre delle "opere sociali", sul tipo di quelle che lo stato protestante moderno svilupperà in seguito ${ }^{14}$.

La preoccupazione sociale fa parte integrale del pensiero teologico di Zwingli. Egli sostiene che:

Sempre la parola di Dio fa menzione dei poveri, viene loro in aiuto, consola gli afflitti e i disperati, mentre coloro che ripongono la loro fiducia in se stessi, Dio li combatte ${ }^{15}$.

Zwingli sviluppa una concezione teologica del povero, inclusi i "poveri in spirito", nella quale la povertà esprime l'essenza della realtà cristiana e, quindi, della società che si vuole tale. La povertà, compresa alla luce della Bibbia, è per lui normativa di ogni vita cristiana. Così, è la povertà, a diventare il fondamento teologico di ogni diaconia cristiana. Non si può essere cristiano in altra maniera che da povero, poiché Cristo e Maria sua madre hanno dimostrato che la condizione cristiana non poteva essere che quella della povertà, sia spirituale che materiale. La povertà spirituale è una virtù, la povertà materiale, invece, non è che l'illustrazione evidente della mancanza di giustizia e di condivisione, di cui gli uomini sulla terra, cristiani inclusi, si rendono colpevoli. Ma questa povertà nel suo insieme è sempre nuovamente rivalorizzata dal fatto che Cristo stesso se ne è rivestito, conferendole il suo significato teologico: mediante la povertà, possiamo vivere secondo l'esempio di Cristo.

L'intera società cristiana è chiamata a organizzarsi e a vivere in conformità a questo fondamento cristologico: da qui scaturisce l'im-

E. Saxer, Huldrych Zwingli, Ausgenwählte Schriften in neuhochdeutscher Wiedergabe mit einer historisch-biographischen Einführung, Neukirchen 1988; G. Hammann, Storia del diaconato. Dal cristianesimo delle origini ai riformatori protestanti del XVI secolo, p. 257.

${ }^{14}$ Cf. G. Hammann, Storia del diaconato. Dal cristianesimo delle origini ai riformatori protestanti del XVI secolo, p. 257.

${ }^{15}$ H. Zwingli, Von Klarheit und Gewissheit des Wortes Gottes, in: Huldreich Zwinglis sämtliche Werke. Corpus Reformatororum LXXXVIII, a cura di E. Egli, G. Finsler, vol I, Berlin 1905, p. 383. 
portanza di questa teologia sociale della diaconia presso il Riformatore di Zurigo.

Nel pensiero di Zwingli è da rilevare il legame tra la diaconia e la mariologia, traccia di un'eredità medievale. Il Riformatore collega la povertà con la figura di Maria, la serva ${ }^{16}$. Per lui, questa è un simbolo privilegiato di povertà, che rinvia a $\mathrm{Cristo}^{17}$. $\mathrm{E}$ in questo senso che egli la elogia a profusione e le attribuisce un posto teologico sorprendente:

Nessuno potrebbe nascere più povero di Cristo, poiché egli non ha nemmeno avuto il diritto a un posto al momento della sua nascita. Questa povertà ci insegna innanzitutto che Dio non colma i suoi preferiti di beni effimeri, ma, mediante l'avversità in questo mondo, mette alla prova la loro fede e la loro speranza [...]. In secondo luogo, noi dobbiamo meditare sul fatto che Dio ha dato al proprio Figlio la povertà in virtù dell'amore per noi, affinché imparassimo sin dalla nostra infanzia ad accettarla, a nostra volta, con serenità, dal momento che il nostro Signore e Salvatore, con sua madre piena di grazia, ha sofferto la povertà e la miseria dalla nascita alla morte ${ }^{18}$.

La povertà della stalla di Betlemme, immagine dell'esistenza di Maria e di suo figlio, è un segno di benedizione, non di maledizione; lo stesso accade per gli uomini. Zwingli non dice questo per esigere dai poveri che si rassegnino alla propria sorte, ma affinché si sentano, nonostante e soprattutto grazie alla loro povertà, amati da Dio più che i benestanti, e affinché questi ultimi si rendano conto dei pericoli inerenti alla loro situazione ${ }^{19}$. Perciò, egli dice,

non abbiate paura di essere poveri con i poveri e miseri con i miseri ${ }^{20}$.

${ }^{16}$ Cf. E. Campi, Via antiqua, umanesimo e Riforma. Zwingli e la Vergine Maria, Torino 1986, pp. 55-56.

${ }_{17}$ Ibidem, p. 56: "Se è certo che nel pensiero di Zwingli Cristo e Maria sono intimamente connessi, è anche vero che la concezione zwingliana di Maria è, nelle sue più profonde radici, in funzione di Cristo, è sistematicamente subordinata alla cristologia e non può prescindere da essa”.

${ }_{18} \mathrm{H}$. Zwingli, Ein predig von der reinen gotzgebärerin Maria, in: ZW I, p. 417.

${ }^{19}$ Il Riformatore tocca qui un tema caro al rinnovamento monastico del XII e XIII secolo, in particolare nel movimento francescano. Alcune sue frasi sulla povertà sono vicine a quelle di Francesco o, più ancora, a quelle di Chiara d’Assisi.

${ }^{20} \mathrm{H}$. Zwingli, Ein predig von der reinen gotzgebärerin Maria, in: ZW I, p. 398. 
È interessante notare questa articolazione fatta da Zwingli tra la preoccupazione diaconale e la teologia mariana. Si sa dell'importanza sorprendente e relativamente poco conosciuta che il Riformatore zurighese accorda a Maria ${ }^{21}$. Ma, deviandone il significato in rapporto a ciò che essa fu nel Medioevo, Zwingli vede in Maria una figura esemplare di umiltà e di povertà, simbolo di vita e di obbedienza pratiche ${ }^{22}$. Si potrebbe dire una figura diaconale. Si comprende così il motivo per cui il Riformatore abbia conservato la maggior parte dei temi mariani tradizionali (salvo quello dell'immacolata concezione), persino quello della verginità perpetua ${ }^{23}$. Si nota tuttavia che è il suo cristocentrismo, d'altronde a somiglianza di Lutero, che gli fa mantenere questa teologia mariana trasformandola in questo significato caritativo: l'incarnazione di Cristo implica la condizione di verginità, di umiltà, di povertà di Maria, che è l'ancilla, la serva per eccellenza. Il suo esempio può avere un altro valore formativo per il credente, sensibilizzandolo alle virtù caritative da lei incarnate. È modello del servizio, perché Cristo è dovuto nascere in questa stessa condizione; è serva, perché Cristo è stato il primo e il più grande dei servi. È dunque proprio perché Cristo è nato e vissuto nella povertà del servizio che la pietà mariana può sensibilizzare la coscienza diaconale dei fedeli ${ }^{24}$. Questo è attestato dalle Scritture e questo fondamento deve sostenere ogni attività riformatrice basata sulle Scritture. Così l'impegno di povertà non potrà essere l'appannaggio di ordini monastici privilegiati, ma il voto di ogni cristiano salvato che Cristo chiama a seguirlo nella spoliazione che egli stesso aveva sperimentato. Questo legame tra diaconia e Maria nel pensiero di Zwingli, questa accentuazione sociale della teologia mariana potrebbe proprio essere originale nella Riforma protestante ${ }^{25}$.

Zwingli non riconosce alla Chiesa, da lui riformata, che un solo ministero, quello della Parola. Su questo piano, egli ha la stessa concezione di Lutero e, per quanto concerne il ministero diaconale, Zwingli classifi-

${ }^{21}$ Cf. W. Tappolet, Das Marienlob der Reformatoren, Tübingen 1962, pp. 221 ss.

22 Cf. H. Düfel, Luthers Stellung zur Marienverehrung, Göttingen 1968.

${ }^{23}$ Cf. E. Campi, Via antiqua, umanesimo e Riforma. Zwingli e la Vergine Maria, pp. 27-53.

${ }^{24}$ Cf. ibidem, pp. 59-91.

${ }^{25}$ Cf. G. Hammann, Storia del diaconato. Dal cristianesimo delle origini ai riformatori protestanti del XVI secolo, p. 261. 
ca tra i ministeri anche i "servi", cioè i diaconi della carità, ossia coloro che distribuiscono elemosine e aiuti ai poveri. Costoro fanno parte dei "ministri della Parola", pur non essendo dei pastori nel senso stretto del termine. La particolarità della concezione zwingliana dei ministeri vuole che i "pastori" comprendano tutti coloro che assumono la "pastorale" del popolo dei fedeli.

Tutti sono "ministri della Parola" nella misura in cui questa Parola rappresenta la proclamazione del Vangelo. Anche coloro che si occupano della diaconia, nel senso caritativo dato da Zwingli, sono perciò dei ministri della Parola, ossia del messaggio evangelico, senza tuttavia essere dei "predicatori". Il loro compito non consiste nell'insegnare, nel senso zwingliano di "profetare" mediante la predicazione orale - senso che si ritrova nella concezione protestante della "predica" o del "sermone" - ma nel "profetare" in azioni, cioè nel predicare la Parola di Dio nella forma concreta del gesto della carità.

I diaconi, perciò, a somiglianza dei servi descritti negli Atti degli Apostoli 6, 1-7, fanno parte del ministero della "Parola"26. Sono dunque, ministri della Parola anche coloro che si prendono cura dei malati, li visitano, gestiscono la solidarietà e provvedono ai bisogni dei poveri. Nel progetto zwingliano costoro assumeranno il compito caritativo verso l'intera società ${ }^{27}$.

Secondo Zwingli il diaconato è un fattore indispensabile all'autentica Chiesa di Gesù Cristo. Il suo fondamento si trova nella Scrittura. Senza servizio diaconale una Chiesa non è più "vera" Chiesa.

Il diaconato ha come scopo e campo d'azione i "poveri e i malati", cioè coloro che sono materialmente sprovvisti. La realtà dei poveri ha un significato teologico: essi vivono la condizione di Cristo stesso nella sua incarnazione, e in questo senso sono i privilegiati, sono i più prossimi a Dio, sullesempio di Maria o dei santi apostoli.

Di conseguenza coloro che sono privi del necessario sono i simboli teologici di tutta la società umana; da qui la necessità di porli non al

${ }^{26}$ Cf. E. A. McKee, John Calvin. On the Diaconate and the Liturgical Almsgiving, Genève 1984, pp. 139-158.

27 Cf. G. Hammann, Storia del diaconato. Dal cristianesimo delle origini ai riformatori protestanti del XVI secolo, p. 274. 
margine, ma nel cuore della società. In questo senso, il diaconato non può essere che sociale e non solamente ecclesiale, cioè appannaggio riservato alle istituzioni di obbedienza clericale.

Se coloro che non possiedono nulla restano poveri, è perché sono stati spogliati dei beni che appartengono a loro, cioè tutto ciò che i possidenti hanno di superfluo. Questo superfluo deve essere necessariamente restituito a loro; proprio questo è lo scopo della diaconia, concepita come servizio caritativo.

Perciò, spetta all'autorità temporale, che ne ha ricevuto la missione da parte di Dio, di adempiere questo compito. Quest'ultimo non sarà più riservato alle persone e istituzioni private, di obbedienza religiosa, ma sarà imposto a tutta la comunità civile. Confiscando i beni immobili del clero e facendosi carico della raccolta dei fondi diaconali presso tutti i fedeli, il magistrato troverà i mezzi materiali per la sua nuova missione caritativa ${ }^{28}$.

Gli esecutori necessari per la sua realizzazione saranno i nuovi "diaconi", nella linea dei diversi sviluppi del ministero della Parola evidenziati da Zwingli nei testi del Nuovo Testamento. Questi diaconi faranno parte dei hirten (pastori) e dei priester (preti), ma con una funzione specifica, quella di "predicare" mediante compiti diaconali caritativi. Secondo il piano di Zwingli, la Chiesa, ossia la società cristiana, ha certamente bisogno di diaconi, ma di diaconi-assistenti sociali.

È in questa prospettiva che Zwingli definisce il ministero di diacono. Nello spazio ecclesiologico e ministeriale, egli lo situa tra i poveri e i preti, come l'intermediario caritativo tra gli indigenti e colui che, nella tradizione della Chiesa, ha sempre ricevuto i fondi ecclesiastici con la missione di ridistribuirli a loro. Nellarea diaconale ci sono dunque, per Zwingli, da una parte i ministri, gli hirten, sinonimo anche di lebrenden (insegnanti) $^{29}$, e i loro diener (diaconi, nel senso di "servi"), dall'altra le pecore, simboleggiate dagli indigenti; i diaconi, invece, sono coloro che realizzano nella pratica l'assunzione dell'incarico caritativo ${ }^{30}$. Ma, dice Zwingli che, questo lavoro pratico di ridistribuzione poteva anche essere

${ }^{28}$ Cf. ibidem, pp. 276-277.

${ }^{29}$ Cf. H. Zwingli, in: ZW III, p. 454: "die lerenden und armen einer yeden klichhöre".

${ }^{30}$ Cf. ibidem, pp. 393-394. 
fatto, nella tradizione, dai priester stessi ${ }^{31}$. Si può constatare, allora, che la fusione tra le responsabilità pastorale e diaconale è del tutto possibile, benché non raccomandabile per il Riformatore zurighese, in particolare nelle parrocchie, così come erano organizzate nel suo tempo.

Secondo G. Hammann, nel progetto diaconale zwingliano la funzione diaconale tradizionale, cioè liturgica appare come una curiosità ormai fuori moda. Infatti, se Zwingli incorpora senza equivoco il diaconato caritativo nella sua concezione del ministero di hirt (o priester), il compito del diaconato sociale così come egli lo concepisce non ha più nulla di liturgico: o è chi assicura il ministero di insegnamento e di culto, il "prete-pastore" (il priester o hirt), che si fa ugualmente carico della diaconia parrocchiale (e allora non cè più un diaconato specifico distinto dal pastorato), o allora sussiste un ministero particolare per la solidarietà, e questo ministero si deve relegare nel suo compito specificamente caritativo e sociale. Zwingli non sembra essersi preoccupato di ricollocare il compito della solidarietà nel cuore del culto domenicale della comunità, per assegnarli un ruolo proprio al centro dello svolgimento liturgico. Egli pare troppo preoccupato di estendere il diaconato all'insieme della società e troppo preoccupato di eliminare il tradizionale clericalismo ministeriale, per immaginare una nuova funzione liturgica diaconale ${ }^{32}$.

\section{GIOVANNI CALVINO (1509-1564)}

Giovanni Calvino propone unarticolazione teologica ed ecclesiologica in base ai tre seguenti attributi del ministero diaconale specificamente ecclesiale: la diaconia caritativa, la sua realtà cultuale e la sua consacrazione ecclesiale. Secondo Calvino non è sufficiente che la solidarietà caritativa ritrovi, in generale, nelle nuove c hiese un posto liturgico sotto la forma di colletta, perché allo stesso tempo appaia anche un ministero cultuale di diacono.

31 Cf. ibidem, p. 394.

32 Cf. G. Hammann, Storia del diaconato. Dal cristianesimo delle origini ai riformatori protestanti del XVI secolo, pp. 278-279. 
Di fatto, anche i pastori, o gli anziani, potrebbero procedere a questa colletta intracultuale e riconsegnarla, successivamente, ai diaconi in qualità di operatori del servizio extracultuale. Si tratterà, dunque, di restituire al diaconato caritativo sia la sua specificità cultuale che la sua qualifica ministeriale propria.

\subsection{IL DIACONATO COME MINISTERO SPECIFICO A FIANCO DEL PASTORATO}

Secondo Calvino è il rigore della ricerca biblica che determina la concezione dei ministeri e, in base al suo biblicismo, il Nuovo Testamento non può essere che normativo per quella concezione della Chiesa Riformata che egli auspica. Tra i testi biblici determinanti per la teologia calviniana della diversità dei ministeri, il Riformatore cita frequentemente le seguenti Lettere dell'Apostolo Paolo: Rm 12, 6-8, 1 Cor 12, 28 ed Ef 4, 11.

Le quattro funzioni di dottore, pastore, anziano e diacono rappresentano il ministero di insieme della Chiesa e sono quattro funzioni indispensabili alla sua missione. Tra questi ministeri specifici, quelli essenziali sono il presbitero, prete o pastore - che riunisce le funzioni ministeriali della Parola e della cura d'anime - e il diacono.

Calvino comincerà a riformare il compito cultuale di ciascuno dei due ministeri. Al pastore spetteranno la predicazione e la presidenza dei sacramenti, compresa l'assoluzione dei peccatori ${ }^{33}$, mentre i fondamenti biblici consentono di restituire al diacono un posto appropriato nello svolgimento liturgico, attribuendogli così una specificità rinnovata.

Seguendo i Riformatori della prima generazione, Calvino riprende dalle Chiese delle origini le caratteristiche originarie del diaconato ministeriale, vi riconosce le norme per la sua restaurazione e critica ciò che è avvenuto in seguito ${ }^{34}$. Dalla fine dell'antichità, secondo lui, la funzione diaconale è stata corrotta nella sua responsabilità caritativa e svilita nel suo ruolo liturgico lungo tutto il Medioevo:

33 Cf. G. Calvino, Institution de la Religion chrestienne, a cura di J. D. Benoît, Paris 1957-1963, vol. III, pp. 13-14, 118-119, 122.

34 Cf. ibidem, p. 77. 
Da parecchie lettere di san Gregorio risulta che nel suo tempo, in cui l'ordinamento della chiesa era molto corrotto, permaneva ancora l'usanza che i diaconi fossero dispensatori dei beni della chiesa sotto l'autorità dei vescovi [...]. L'esser ordinati per leggere l'Evangelo, per esortare il popolo a pregare e per porgere al popolo il calice nella Cena era per onorare il loro stato, affinché compissero il loro dovere con più grande timor di Dio e da tali cerimonie fossero ammoniti che essi non si trovavano in una condizione profana, ma in un incarico spirituale e consacrato a $\mathrm{Dio}^{35}$.

Calvino contesta che il compito del diacono sia quello di "giocare" al pastore; egli deve avere una funzione ministeriale specifica che il pastore non può esercitare.

Nel sermone su 1 Tm 3, 8-10 Calvino afferma:

Ora, perché i papisti istituiscono i diaconi? È per recitare una farsa, proprio come dei ciarlatani. Infatti, quelli che sono fatti diaconi e suddiaconi nel papato, lo sono forse per aver cura dei poveri, e per distribuire le elemosine? No, ma nella messa essi saranno là vestiti e giocheranno a fare il prete. E hanno recitato una tale farsa? Li si fa preti, in seguito [sul campo]. E non è forse troppo per burlarsi di Dio? Poiché san Paolo non ha pensato che i diaconi dovessero essere dei pastori di chiesa, ne siamo ben lontani. Un uomo che sarà adatto all'ufficio di pastore, non potrà essere diacono, e non sarà idoneo a distribuire le elemosine. $\mathrm{E}$ ugualmente nel caso opposto: ve ne saranno molti che potranno avere la cura dei poveri, che non saranno affatto [idonei] per insegnare. È dunque necessario conoscere ciò che Dio ha dato a ciascuno ${ }^{36}$.

Respingendo la collocazione cultuale che il diacono ordinato aveva nella Chiesa medievale, Calvino vuole evitare che il ministero di diacono sia ridotto a un sotto-pastorato. Egli vuole restituire al diaconato la sua specificità ministeriale. Dio ispira vocazioni differenti, a seconda che egli chiami dei pastori o dei diaconi; le loro funzioni ministeriali non sono intercambiabili. Calvino è molto chiaro sulla distinzione da fare tra i due ministeri. Il diaconato deve avere la sua specificità nel ruolo propriamente

${ }^{35}$ Ibidem.

${ }^{36}$ G. Calvino, Sermoni sulla Prima lettera a Timoteo, in: Ioannis Calvini Opera quae supersunt omnia, a cura di G. Baum, E. Cunitz, E. Reuss, Corpus Reformatorum XXIX-LXXXVII, Brunsvigae 1863-1900, LIII, col. 303. 
caritativo. Il posto cultuale del diacono dovrà restare dunque in questa specificità, e non slittare verso una mini-funzione pastorale.

\subsection{IL DIACONATO COME UN MINISTERO PERMANENTE E ORDINATO}

Per Calvino, il ministero di diacono praticato nel suo tempo nella Chiesa stabilita, non è più conforme a quello delle Chiese antiche, ma è stato deformato e spogliato della sua ecclesialità, della sua consacrazione:

Riguardo allordine dei diaconi, noi saremmo del tutto d'accordo se quest'ufficio fosse restituito alla sua integrale purezza, quale esso ha avuto sotto gli apostoli e nella chiesa antica. Ma i diaconi fabbricati da questa gente, che cosa hanno di simile con quelli? Io non parlo affatto delle persone, perché non si lamentino di essere ingiuriate perché la loro dottrina è stimata sulla base dei vizi delle persone; ritengo però che essi agiscono in maniera irrazionale quando per il loro diaconi (così come ce li descrivono nella loro dottrina) fan riferimento a coloro che furono ordinati dalla chiesa apostolica. Affermano che ai loro diaconi spetta di assistere i preti, e di provvedere tutto ciò che è richiesto nei sacramenti, per esempio nel battesimo e nella cresima, di versare il vino nel calice e mettere il pane sulla patena, ordinare con proprietà l'altare, portare la croce, leggere al popolo l'Evangelo e l'epistola. Cè forse in tutto questo una sola parola del vero ufficio dei diaconi? ${ }^{37}$.

Secondo Calvino, il modo di consacrare questi "cosiddetti" diaconi non è che "farsa e commedia", cose che gli apostoli non avrebbero mai riconosciuto come autenticamente ecclesiali ${ }^{38}$. Occorre, dunque, riformare e restaurare l'originario ministero dei tempi apostolici, unica possibilità per restituirgli la sua ecclesialità, la sua consacrazione mediante lo Spirito Santo e la sua efficacia ministeriale.

Calvino chiede espressamente che il diaconato sia considerato come un ministero ecclesiale e non soltanto sociale. Lo dice nello stesso sermone sulla Prima lettera a Timoteo:

${ }^{37}$ Idem, Institution de la Religion chrestienne, vol. IV, pp. 499-500.

38 Cf. ibidem, p. 500. 
Confermiamo ciò che qui è dichiarato, che cioè tutti coloro che sono eletti per distribuire le elemosine e per governare il pane dei poveri, non soltanto sono nello stesso stato di [persona] pubblica, ma appartengono al regime spirituale della chiesa, e sono come officiali di Dio, al fine di distribuire i sacrifici che gli sono offerti e consacrati. Poiché egli se ne vuole servire in una condizione così onorevole, è ben a ragione che, da parte loro, essi vedano di camminare con timore, come chi ha da rendere conto a un troppo grande signore. E come coloro che sono chiamati a questa carica devono essere istruiti dall'ammonizione di san Paolo di comportarsi lealmente, così quando li si elegge, si proceda rispettando ciò che egli comanda qui, se non ci si voglia trovare colpevoli di aver pervertito l'ordine della chiesa nell'ultimo giorno. Poiché se vogliamo che ci sia chiesa tra noi, bisogna che manteniamo quel regime che Dio ha stabilito come inviolabile ${ }^{39}$.

Se si vuole "che ci sia chiesa tra noi", secondo Calvino, è necessario che si ristabilisce questo ordine di Dio riconosciuto come inviolabile! Il ministero ecclesiale e permanente di diacono, distinto dal pastorato, per lui è dunque proprio indispensabile per ogni autentica Chiesa, poiché questo ordine è stabilito da Dio, ed è un ministero permanente ${ }^{40}$.

Fondato sulla Bibbia, il ministero diaconale riceve la sua giustificazione teologica. Calvino vuole spiegare persino perché un ministero così importante come quello di diacono non sia stato istituito secondo At $6,1-7$, che in un secondo tempo ${ }^{41}$. Il motivo, per Calvino, consiste nel fatto che gli apostoli erano esposti a pressioni contestuali così forti che non potevano più assumere questo compito; di qui la necessità ispirata dallo Spirito Santo di eleggere dei ministri complementari ${ }^{42}$. Questa è la dimostrazione, molto esemplare, che la Chiesa si istituisce in modo progressivo ma non perciò meno autentico, e che lo Spirito Santo può trasformare una situazione ecclesiale di tensione in unoccasione di perfezionamento. È la situazione di indigenza divenuta insopportabile che ha

${ }^{39}$ G. Calvino, Sermoni sulla Prima lettera a Timoteo, in: CO LIII, coll. 292-294.

${ }^{40}$ Cf. E.A. McKee, John Calvin. On the Diaconate and the Liturgical Almsgiving, pp. 153-158.

${ }^{41}$ Cf. ibidem, pp. 139-153.

${ }^{42}$ Cf. G. Calvino, Commentarius in Acta Apostolorum, in: CO XLVIII, coll. $117-118$. 
stimolato i discepoli e gli apostoli a organizzare meglio la loro comunità, affinché nel mondo e nonostante le sue miserie, la Chiesa possa adempire la sua missione. Una situazione puramente temporale prende così il suo significato spirituale e diventa lo strumento dell'azione divina nella Chiesa e al centro della storia umana.

L'episodio dell'elezione dei sette "diaconi" secondo il testo di Atti ${ }^{43}$ non può giustificare la riduzione del diaconato a una semplice funzione temporale di assistenza sociale organizzata dall'autorità temporale. Deve esserci un ministero diaconale che da una parte dipenda dall'autorità ecclesiale e che, dall'altra, non sia un sotto-pastorato, ma un ministero specifico, distinto dagli altri, compreso quello del pastorato. Un ministero dai compiti senzaltro differenti da quelli del pastorato, ma permanente e ordinato come quest'ultimo. Calvino lo ripete spesso in differenti occasioni:

Resta da trattare [...] la cerimonia dellordinazione. Risulta evidente che gli apostoli non ne ebbero altra oltre l'imposizione delle mani [...]. Comunque sia, essi si sono avvalsi di questa cerimonia solenne tutte le volte in cui hanno ordinato qualcuno al ministero della chiesa, come constatiamo dei pastori, quanto dei dottori e dei diaconi. Ora, per quanto non ci sia nessun comandamento specifico a riguardo dell'imposizione delle mani, tuttavia, poiché vediamo che gli apostoli l'hanno costantemente impiegata, ciò che essi hanno osservato tanto diligentemente ci deve valere come fosse un precetto. È certo cosa utile onorare davanti al popolo la dignità del ministero con una tale cerimonia, e in tal modo anche ricordare a colui che è ordinato, che egli non appartiene più a se stesso, ma è consacrato al servizio di Dio e della chiesa [...]. Risulta ben chiaro che questo fu fatto per i sette diaconi, per Paolo e Barnaba, e per alcuni altri $(\text { At } 6,6)^{44}$.

Restituendogli il suo attributo di ministero consacrato, Calvino rende così al diaconato, in modo incontestabile, i suoi attributi ministeriali propri e la sua qualificazione specificamente ecclesiale.

${ }^{43}$ Cf. E.A. McKee, John Calvin. On the Diaconate and the Liturgical Almsgiving, pp. 149-153.

${ }^{44}$ G. Calvino, Institution de la Religion chrestienne, vol. IV, pp. 69-71. 


\subsection{UN MINISTERO CARITATIVO E SOCIALE}

Secondo Calvino, il ministero di diacono deve custodire la sua specificità caritativa in seno alla città. Nonostante gli attributi confessati che potranno essere suoi, il suo campo d'attività è determinato dalla dimensione multitudinista della Chiesa. La sua funzione caritativa si manifesta sotto due distinte forme professionali che sono presenti a $\mathrm{Gi}$ nevra ancor prima dell'arrivo del Riformatore nel 1536: quella del diacono amministratore dei beni ecclesiastici e quella del diacono ospitaliere, una sorta di risorgiva del "quarto ufficio" dei monasteri medievali ${ }^{45}$. Di nuovo, Calvino ricorre ai testi neotestamentari, particolarmente a $\mathrm{Rm} \mathrm{12,8,} \mathrm{per}$ dare fondamento a questi due compiti:

La cura dei poveri è stata affidata ai diaconi, secondo quelle due modalità indicate da Paolo nella Lettera ai Romani: «colui che distribuisce lo faccia con semplicità, colui che esercita la misericordia lo faccia con gioia» $(\mathrm{Rm} 12,8)$. Considerato che egli parla degli uffici pubblici della chiesa, devono esserci stati due tipi differenti di diaconi. Ora, se proprio non m’inganno, nel primo caso egli descrive i diaconi che distribuivano le elemosine, nel secondo quelli che avevano l'incarico di provvedere ai poveri e servirli $[\ldots]$. Se noi accogliamo questa tesi, così comè giusto che sia, visto che è fondata su valide motivazioni, vi saranno due tipi di diaconi: i primi serviranno la chiesa, governando e dispensando i beni ai poveri; i secondi, servendo i malati e gli altri poveri ${ }^{46}$.

Su questo punto Calvino non è originale, se non per la nitidezza delle sue affermazioni e per le loro implicazioni sul piano istituzionale. Egli ripropone la linea caritativa della tradizione antica e medievale con la pretesa di restituirle il suo fondamento biblico prioritario. La sua posizione di base è quella degli altri Riformatori ${ }^{47}$.

${ }^{45}$ Cf. Ph. Ariès, G. Duby, Histoire de la vie privée, Paris 1985-1987, vol. II, pp. 61 ss.

${ }^{46}$ G. Calvino, Institution de la Religion chrestienne, vol. IV, p. 63.

${ }^{47}$ Cf. B. Pasquier, John Knox et la Réformation écossaise, Neuchâtel 1993, pp. $76-78$. 
Secondo G. Hammann ${ }^{48}$ l'originalità del Riformatore ginevrino si manifesta nella sua volontà di restituire a questa duplice funzione caritativa una priorità assoluta:

Ora, nonostante che il nome di diaconia abbia un significato assai più ampio, tuttavia la Scrittura definisce diaconi specialmente coloro che dalla chiesa sono costituiti per distribuire le elemosine e sono come esattori e procuratori dei poveri, la cui origine, istituzione e incarico sono descritti da san Luca negli Atti (At 6, 3). Infatti tra i greci era sorta una lamentela, per il fatto che non si teneva conto delle loro vedove nella distribuzione dei doni ai poveri; gli apostoli, scusandosi per non poter soddisfare a due uffici, cioè la predicazione e la cura dei poveri, chiedono al popolo di eleggere sette uomini di buona fama, che abbiano questo incarico. Questi sono stati i diaconi del tempo degli apostoli, e tali anche noi dobbiamo avere, sullesempio della chiesa delle origini ${ }^{49}$.

È proprio questa duplice funzione che il Riformatore ginevrino cercherà di reintrodurre nella pratica della sua Chiesa.

Esaminiamo i diaconi e la distribuzione dei beni da loro praticata. In realtà i loro diaconi non sono creati per questo; infatti non pretendono da loro altro se sono servire l'altare, cantare l'Evangelo e non so quali altre cose inutili ${ }^{50}$.

\subsection{UN MINISTERO LITURGICO}

Attraverso il ritorno alle origini e grazie alla sua notevole conoscenza dei Padri e dei canoni ${ }^{51}$ della Chiesa, Calvino vuole riscoprire gli autentici attributi liturgici del diaconato. Egli nella sua critica sarcastica nei confronti dell'ufficio dei diaconi durante la Messa, già segnalata, richiama puntualmente il loro l'autentico servizio cultuale, così come era un tempo:

${ }^{48}$ Cf. G. Hammann, Storia del diaconato. Dal cristianesimo delle origini ai riformatori protestanti del XVI secolo, p. 330.

${ }^{49}$ G. Calvino, Institution de la Religion chrestienne, vol. IV, pp. 63-64.

50 Ibidem, p. 100.

${ }^{51}$ Cf. L. Grane, A. Schnidler, M. Wriedt, Auctoritas Patrum, I-II. Zur Rezeption der Kirchenväter im 15. und 16. Jahrhundert, Mainz 1993. 
Cề un punto nella messa in cui costoro che recitano la parte di diaconi rappresentano farsescamente l'antica tradizione quando ricevono le offerte che si fanno prima della consacrazione. Ora, la celebrazione antica voleva che i fedeli, prima di comunicare nella Cena, si baciassero l'un con l'altro e poi offrissero le loro elemosine sull'altare. Così rendevano testimonianza della loro carità, in primo luogo con un segno, e poi con unazione. Il diacono, che era procuratore dei poveri, riceveva ciò che era offerto, per distribuirlo. Attualmente, di tutte queste elemosine, ai poveri non giunge un solo quattrino, proprio come se venissero gettate in fondo al mare. Perciò quelli si prendono gioco della chiesa con questa copertura menzognera, di cui si servono nell'ufficio di diaconi. Certo, in questo diaconato non cè nulla a che vedere con l'istituzione degli apostoli, né con l'antico uso ${ }^{52}$.

È Calvino, nello stesso passo dell'Instituzione, ricorda inoltre che invece di lasciare la loro parte ai poveri come era prescritto dal diritto canonico, i chierici,

come i briganti, dopo aver sgozzato i poveri passanti, dividono tra loro il bottino, così questi uomini di valore, dopo aver spento la luce della parola di Dio, avendo, per così dire, tagliata la gola alla chiesa, hanno ritenuto che tutto ciò che era consacrato per un uso santo fosse consegnato loro come preda e rapina. Pertanto, facendone le parti, ciascuno ha rubato quanto ha potuto. E così tutta la prassi antica non è stata soltanto cambiata, ma interamente capovolta [...]. E questo ordine non cè più tra loro, visto che la distribuzione dei beni ecclesiastici è apertamente trasformata da loro in una ruberia malvagia e sacrilega ${ }^{53}$.

Così, secondo Calvino, la Chiesa ha perduto la sua duplice missione apostolica, la proclamazione dell'Evangelo e la sua traduzione caritativa, cessando così di essere la Chiesa autenticamente cristiana.

Se essa vuol tornare a esserlo, sarà necessario restaurare l'autentica funzione liturgica del diacono. Calvino ne traccia il percorso con la sua spiegazione del testo di At 2, 42-44: è qui, secondo lui, che si trova la congiunzione tra la vita cultuale della comunità ecclesiale e la sua realtà quotidiana:

${ }^{52}$ G. Calvino, Institution de la Religion chrestienne, vol. IV, pp. 100-101.

53 Ibidem, pp. 101-102. 
E pertanto dobbiamo riflettere bene su questo passo, poiché non è affatto senza motivo che san Luca ha unito queste due parole, la Cena e la comunione, per mostrarci che dobbiamo stabilire tra noi una vera unione, una carità fraterna per comunicare gli uni con gli altri, considerando che Dio ci ha fatti gli uni per gli altri come membra di uno solo corpo, che è Gesù Cristo. E infatti durante la Cena (io parlo del tempo in cui la si celebrava più spesso di quanto lo si faccia ora), per testimoniare la carità fraterna che noi dobbiamo avere gli uni con gli altri, ci si baciava gli uni con gli altri. E poi c'erano le elemosine per sostenere coloro che ne avevano necessità; con questo si dimostrava che non era per pura fantasia che ci si chiamava fratelli. E questo era proprio insito nella cerimonia come tutto il resto. Infatti, dal momento che siano tutti membra di Cristo, ritenevano che se alcuni si fossero trovati nell'indigenza era come se fossero loro a soffrire per la mancanza nel corpo di Cristo. E pertanto donavano denaro e facevano elemosine per venire incontro al bisogno. Ma tutte queste cose sono state corrotte durante il papato [...]. Perciò noi dobbiamo attenerci alla vera regola che riceviamo dalla dottrina degli apostoli, ossia di essere uniti insieme come membra di un solo corpo, di una vera unione e carità fraterna, comunicando gli uni gli altri in ciò di cui saremo capaci, se vogliamo che Dio approvi le nostre assemblee e la comunione a questi sacramenti ${ }^{54}$.

Calvino vede, nei quattro elementi citati da Luca (l'insegnamento, la comunione fraterna, la frazione del pane e le preghiere), le quattro caratteristiche di ogni pratica ministeriale nella Chiesa. Mediante la "comunione fraterna" e la "frazione del pane", la diaconia trova il suo inserimento liturgico, nella spartizione dei beni e nel servizio agli indigenti. Il luogo d'inserimento che Calvino ritrova, grazie a questo testo, per il ministero diaconale in quanto espressione della carità è quello della liturgia eucaristica. Egli si collega così alla pratica delle Chiese delle origini e di quelle antiche. Fa notare però che non bisogna concepire la "comunione fraterna" nel senso stretto che l'eucaristia ha assunto in seguito, ma precisamente nel senso di una condivisione di vita quale è illustrata dai pasti comunitari delle prime Chiese:

«Spezzando il pane nelle case». San Luca indica che essi non soltanto mostrarono in pubblico dei segni di vera religione, ma anche che tenevano

${ }^{54}$ E.A. McKee, John Calvin. On the Diaconate and the Liturgical Almsgiving, p. 86. 
uno stesso comportamento nella loro vita privata. Quanto al fatto che alcuni in questo passo interpretano lo spezzare del pane come la santa cena, mi sembra lontano dall'intenzione di Luca. Significa dunque che erano soliti prendere i loro pasti insieme e sobriamente. Infatti coloro che fanno dei banchetti sontuosi, non mangiano così familiarmente insieme. Egli aggiunge subito dopo "in semplicità di cuore", il che è anche segno di temperanza. Insomma, egli vuol dire che il loro modo di vivere era sobrio e fraterno. Alcuni collegano la gioia e la semplicità di cuore con la lode a Dio. E questo può essere senz’altro accolto. Ma perché lodando Dio non può esserci la semplicità di cuore se non si mostra in tutte le parti della vita: per questo motivo è certo che nel testo si parla di quella "semplicità", poiché i fedeli la dimostravano in tutto e per tutto [...]. Ma se qui la colleghiamo con la frazione del pane, ciò significherà anche amore genuino e puro, quello in cui i cuori si aprono l'uno all'altro e nessuno cerca con astuzia il proprio profitto particolare ${ }^{55}$.

Calvino vuole restituire l'unità tra la realtà spirituale - cultuale, e la realtà materiale - quotidiana, dei fedeli. I beni materiali, nella fattispecie il campo di attività dei diaconi, non devono essere minimizzati sul piano spirituale; prova ne sia la maniera in cui i primi cristiani hanno voluto e saputo integrare tutta la loro realtà quotidiana nel cuore del culto e delle sue caratteristiche fondamentali. Mediante questo suo reinserimento liturgico, Calvino vuole a restituire al ministero diaconale la sua qualificazione spirituale. La carità, egli dice, è preghiera; le Chiese antiche lo attestano:

Per quanto concerne la chiesa antica, i diaconi avevano la cura di tutto ciò che le apparteneva, e tutto ciò che era donato alla chiesa era affidato a loro, e noi vediamo come era strettamente raccomandato loro di camminare come davanti a Dio, di considerare che non si trovavano affatto in un ufficio profano né mondano, ma che questo era un incarico spirituale, e proprio per questo motivo si permetteva che porgessero il calice durante la celebrazione della Cena di nostro Signore Gesù Cristo; quelli che avevano l'incarico di aver cura dei poveri si trovavano, infatti, insieme ai ministri della parola di $\mathrm{Dio}^{56}$.

${ }^{55}$ G. Calvino, Le premier livre des commentaires de M. Jean Calvin, sur les Actes des Apostres, Genève 1552, pp. 53-58.

${ }^{56}$ E.A. McKee, John Calvin. On the Diaconate and the Liturgical Almsgiving, p. 154. 
Praticare la diaconia, mettersi al servizio della sventura dei più indigenti, è servire Cristo e lodare Dio. Ora, questa lode e questo servizio trovano la loro espressione più adeguata nel culto domenicale, secondo la descrizione del libro degli Atti. Preghiera e carità sono i sacrifici profumati del culto cristiano ${ }^{57}$. Dove è celebrato Cristo, anche la carità - ossia la pratica diaconale - deve essere ugualmente presente, e con preferenza verso le persone deboli e verso i poveri. In tal modo essa sarà generatrice di concordia, di pace, di giustizia e di unità tra tutti, compresi i più bisognosi. Il suo significato e il suo effetto sono tali, nella liturgia, che nessuna Chiesa autentica potrebbe farne a meno nel luogo e nel momento della propria celebrazione comunitaria. Qualora ne facesse a meno, come accadde dalla fine dell'antichità sino a tempo odierno, non può che perderne di sostanza e di impatto sulla realtà quotidiana, pubblica e privata. Mediante il diaconato e il suo ruolo liturgico, in particolare eucaristico, la comunità confessante deve aprirsi al mondo e ai suoi problemi, come predicazione vivente della Parola-carità, testimonianza essenziale e opera spirituale verso tutta l'umanità.

\section{CONCLUSIONE}

Per i Riformatori più importanti della Chiesa protestante del XVI secolo, come Lutero, Zwingli e Calvino l'identità e il ministero del diacono sono di importanza secondaria nella loro riflessione teologica. Lutero attribuisce al diaconato un fondamento specifico, ma quanto a importanza teologica lo subordina al ministero della Parola. Il diaconato è sottostante a quest'ultimo. Nel suo fondamento, dunque, il ministero diaconale è ben distinto da quello della Parola, il solo essenziale alla specificità evangelica della Chiesa. Zwingli tra i ministeri elenca anche i "servi", cioè i diaconi. Il loro compito non consiste nel "profetare" mediante la predicazione orale, ma in azioni, cioè nel predicare la Parola di Dio nella forma concreta del gesto della carità. Secondo il Calvino il ministero ecclesiale e permanente di diacono, distinto dal pastorato è indispensabile per ogni autentica Chiesa, poiché questo ordine è stabilito da Dio, ed è un ministero permanente.

${ }^{57}$ Cf. ibidem, pp. 244-245. 


\section{BIBLIOGRAFIA}

Ariès Ph, G. Duby G., Histoire de la vie privée, Paris 1985-1987.

Bernoulli W., Von der reformierten Diakonie der Reformationszeit, in: Das diakonische Amt der Kirche, a cura di H. Krimm, Stuttgart 1965², pp. 197-202.

Calvino G., Ioannis Calvini Opera quae supersunt omnia, a cura di G. Baum, E. Cunitz, E. Reuss, Corpus Reformatorum XXIX-LXXXVII, Brunsvigae 1863-1900.

Calvino G., Institution de la Religion chrestienne, libri I-IV, a cura di J. D. Benoît, Paris 1957-1963.

Campi E., Via antiqua, umanesimo e Riforma. Zwingli e la Vergine Maria, Torino 1986.

Denzler A., Geschichte des Armenwesens im Kanton Zürich im 16. und 17. Jahrhundert, Zürich 1929.

Düfel H., Luthers Stellung zur Marienverehrung, Göttingen 1968.

Grane L, Schnidler A, Wriedt M., Auctoritas Patrum, I-II. Zur Rezeption der Kirchenväter im 15. und 16. Jahrhundert, Mainz 1993.

Hamm B., Zwinglis Reformation der Freiheit, Neukirchen 1988.

Hammann G., Storia del diaconato. Dal cristianesimo delle origini ai riformatori protestanti del XVI secolo, Magnano 2004.

Köhler W., Armenpflege und Wohltätigkeit in Zürich zur Zeit Ulrich Zwinglis, Zürich 1919.

Lienhard M., Laction et la doctrine de Huldrych Zwingli, in: Histoire du christianisme, a cura di G. Livet, F. Rapp, vol. VII, Strasbourg 1982, pp. 771-787.

Locher G.W., Die Zwinglische Reformation im Rahmen der europäischen Kirchengeschichte, Göttingen-Zürich 1979.

Luther M., Werke. Kritische Gesamtausgabe, Weimar 1883.

Luther M., Oeuvres, Genève 1957.

McKee E.A., John Calvin. On the Diaconate and the Liturgical Almsgiving, Genève 1984.

Meroni P., Il diacono. Segno di rinnovamento della Chiesa. Presenza nella famiglia e nella vita della società, Roma 2010.

Miegge G., Lutero. L'uomo e il pensiero fino alla Dieta di Worms, Torino 2001.

Pasquier B., John Knox et la Réformation écossaise, Neuchâtel 1993.

Saxer E., Huldrych Zwingli, Ausgenwählte Schriften in neuhochdeutscher Wiedergabe mit einer historisch-biographischen Einführung, Neukirchen 1988.

Selejdak R., Diakonat stały w świetle dokumentów Soboru Watykańskiego II, posoborowego Urzędu Nauczycielskiego Kościoła i narodowych «Rationes institutionis diaconorum permanentium», Warszawa 2010.

Tappolet W., Das Marienlob der Reformatoren, Tübingen 1962.

Zwingli H., Huldreich Zwinglis sämtliche Werke. Corpus Reformatorum LXXXVIII ss., a cura di E. Egli, G. Finsler, Berlin 1905. 